Hautarzt 2014 • 65:1020-1021

DOI 10.1007/s00105-014-3543-3

Online publiziert: 13. November 2014

๑) Springer-Verlag Berlin Heidelberg 2014

\author{
Henning Hamm ${ }^{1} \cdot$ Hans Wolff $^{2}$ \\ ${ }^{1}$ Klinik und Poliklinik für Dermatologie, Venerologie und Allergologie, Universitätsklinikum Würzburg, \\ Würzburg, Deutschland \\ ${ }^{2}$ Klinik und Poliklinik für Dermatologie und Allergologie, Klinikum der Universität München, \\ München, Deutschland
}

\title{
Blickpunkt Kopfhaut
}

Liebe Kolleginnen und Kollegen!

Die Kopfhaut ist eine Region des menschlichen Körpers, die verschiedene Eigenheiten aufweist. So ist es das besondere talgreiche Mikromilieu einer stark behaarten Kopfhaut, das Entzündungen durch gering pathogene Hefepilze begünstigt. Die Rede ist von Malassezia spp. und vom seborrhoischen Ekzem. Im Gegensatz dazu prädestiniert das präpubertär talgarme Mikromilieu der Kopfhaut zur Infektion durch Fadenpilze, zur Tinea capitis. Die Kopflaus ist derart adaptiert an das menschliche Kopfhaar, dass sie in keiner anderen Körperregion als am behaarten Kopf und auf keiner anderen Spezies als dem Menschen überleben kann. Dagegen spielt die haarlose Kopfhaut von Glatzenträgern eine bedeutsame Rolle als Sonnenterrasse, auf der sich im höheren Alter oft aktinische Präkanzerosen, Plattenepithelkarzinome und andere durch UV-Licht induzierte maligne Hauttumore entwickeln. An diesen wenigen Beispielen wird deutlich, dass es sich lohnt, diesem speziellen "Territorium“ des Integuments einmal besondere Aufmerksamkeit zu schenken und in einem Leitthemenheft solche dermatologische Erkrankungen zu beleuchten, die sich besonders gerne an der Kopfhaut manifestieren.

Im ersten, kinderdermatologisch geprägten Beitrag stellen Valeria Behle und Henning Hamm Fehlbildungen und Nävi des behaarten Kopfes dar. Dermatologen und Pädiatern sollte bekannt sein, dass ein haarloses Areal mit einem schmalen Ring kräftiger Mehrbehaarung auf eine okkulte kraniale Dysraphie hinweisen kann, was besondere Vorsicht vor operativen Absichten erfordert. Der Naevus sebaceus ist im Gegensatz zu früherer Annahme nicht zwingend entfernungsbedürftig, da sich in ihm im Lauf des Lebens überwiegend gutartige Adnextumoren ausbilden. Basalzellkarzinome und andere maligne epitheliale Tumore stellen eher die Ausnahme dar, obwohl viele Kasuistiken der Fachliteratur das Gegenteil vermuten lassen. Andererseits kann ein Naevus sebaceus durch seine Haarlosigkeit stark stigmatisierend wirken. Daher ist eine vorausschauende operative Entfernung im Kleinkindesalter oft wesentlich günstiger und technisch einfacher durchzuführen als im höheren Lebensalter. Kongenitale melanozytäre Nävi gehen an der Kopfhaut häufiger als in anderen Körperregionen nach einigen Jahren in klinische Regression, weshalb aufwendige operative Maßnahmen reiflich zu überlegen sind. Andererseits sollte bei blauen Nävi und auffälligen erworbenen melanozytären Nävi lieber eine Exzision durchgeführt werden, da Verlaufskontrollen aufgrund starker Behaarung erschwert sind.

\section{》) Die Kopfhaut ist prädestiniert für die verschiedensten dermatologischen Erkrankungen}

Christian Rose beschreibt die Besonderheiten von typischen Neoplasien des Capillitiums. Das maligne Melanom hat am behaarten Kopf eine ungünstigere Prognose als an anderen Körperstellen. Dies liegt nicht nur daran, dass sie durch die Behaarung verdeckt und deswegen später erkannt werden. Auch Angiosarkome werden oft erst bei relativ großer Ausdehnung und Übergreifen auf die nicht behaarte Stirnhaut diagnostiziert. Die Kopfhaut gehört zu den bevorzugten Manifestationsorten von Hautmetastasen innerlicher Tumore, ist aber auch eine Vorzugs- lokalisation gutartiger Adnextumore, wobei hier interessanterweise nicht diejenigen mit follikulärer Differenzierung überwiegen.

Bei vielen Tumoren der Kopfhaut besteht die Therapie der Wahl in der Exzision, die einen mehr oder weniger großen und tiefen Defekt bei geringer Deckungsreserve hinterlässt. Thematisch an den Beitrag über Hauttumoren anschlieBend, stellen Wolfgang Koenen und Christian Kunte daher spezifische Aspekte der Dermatochirurgie am Capillitium dar. So ist es der speziellen Anatomie des Capillitiums geschuldet, dass je nach Größe und Tiefe eines Weichteildefektes unterschiedliche operative Lösungswege gesucht werden müssen. Kleinere und oberflächliche Operationswunden können der Sekundärheilung überlassen oder direkt verschlossen werden. Tiefere Defekte bedürfen dagegen der Versorgung durch Lappenplastiken oder Hauttransplantationen. Periostdefekte mit exponiertem Schädelknochen können durch Bohrlochfenestrationen zur Tabula externa gut zur sekundären Defektdeckung konditioniert werden. Auch die Vakuumversiegelung ist eine Option, die Wundgrundkonditionierung zu befördern.

Dagmar Wilsmann-Theis und Thomas Bieber stellen die Problematik von Ekzemen und der Psoriasis an der Kopfhaut dar. Entzündungen sind in dieser Region für betroffene Patienten nicht nur aufgrund der exponierten Lage kosmetisch belastend, sondern gehen hier auch oft mit einem stärker ausgeprägten Juckreiz als an anderen Körperstellen einher. Bei der Abgrenzung einer Psoriasis von den unterschiedlichen Ekzemformen des Capillitiums ist die Untersuchung des restlichen Integuments hilfreich. Der isolierte Befall der Kopfhaut kann auf ein toxisch- 
irritatives Ekzem durch Haarpflegemittel oder Haarfärbemittel hinweisen, während andere Ekzeme ebenso wie die Psoriasis meist auch Veränderungen in der Umgebung oder an anderen Körperstellen hervorrufen. Therapeutisch kommen aufgrund der meist dichten Behaarung insbesondere Lösungen, Gele und speziell für die Kopfhaut entwickelte Schäume zur Anwendung. Die Psoriasis und die meisten Ekzemformen sprechen dabei gut auf topische Kortikosteroide an. Eine positive Eigenschaft der Kopfhaut besteht darin, dass im Gegensatz zu anderen Körperstellen Nebenwirkungen durch Kortikosteroide erst spät eintreten. Ausgeschlossen sind sie allerdings nicht; eine monatelange Anwendung hochpotenter Kortikosteroide kann durchaus auch an der Kopfhaut zu Teleangiektasien oder Atrophie führen.

Im letzten Beitrag beschreibt Dietrich Abeck Infektionen und Ektoparasitosen, die häufig am Capillitium vorkommen. An erster Stelle ist hier die Pediculosis capitis zu nennen, die nach dem Schnupfen die häufigste infektiöse Erkrankung des Kindesalters darstellt. Seit einigen Jahren steht uns mit Dimeticon-haltigen Präparaten eine neue, sehr effektive Behandlungsmöglichkeit zur Verfügung, die im Gegensatz zu den meisten Alternativen auf einem physikalischen Wirkprinzip ohne absehbares Risiko einer Resistenzentwicklung beruht. Bei der Tinea capitis ist unbedingt auch ein kultureller Erregernachweis zu führen, um die richtige Wahl des oralen Antimykotikums zu treffen bzw. zu bestätigen. Dies ist umso wichtiger, als alle neueren Systemtherapeutika bei Kindern mit Tinea nur „off-label“ eingesetzt werden können und dem behandelnden Arzt bei deren Einsatz eine besonders große Verantwortung zukommt. Lediglich Griseofulvin ist im Kindesalter zugelassen, muss aber oft doppelt so lange eingenommen werden wie die neueren Substanzen. Wichtig sind auch eine antimykotische Lokaltherapie, um die Ansteckungsfähigkeit des Patienten rasch zu verringern, sowie die gleichzeitige Mitbehandlung betroffener menschlicher und nicht selten auch tierischer Familienmitglieder.

Nun hoffen wir, Ihr Interesse für unser Leitthema geweckt zu haben, und wünschen Ihnen viel Vergnügen und neue Er- kenntnisse bei der Lektüre dieses KopfHautarzt-Heftes!

Herzlichst

Ihre

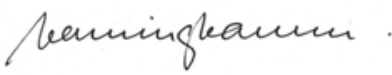

Prof. Dr. Henning Hamm<smiles>N#CCCCCC#N</smiles>

Prof. Dr. Hans Wolff

\section{Korrespondenzadressen}

\section{Prof. Dr. H. Hamm}

Klinik und Poliklinik für Dermatologie

Venerologie und Allergologie

Universitätsklinikum Würzburg

Josef-Schneider-Str. 2, 97080 Würzburg

hamm_h@ukw.de

\section{Prof. Dr. H. Wolff}

Klinik und Poliklinik für Dermatologie und Allergologie, Klinikum der Universität München Frauenlobstraße 9-11, 80337 München Hans.Wolff@med.uni-muenchen.de

\section{Einhaltung ethischer Richtlinien}

Interessenkonflikt. H. Hamm und H. Wolff geben an, dass kein Interessenkonflikt besteht.

\section{Zwei Forschungsvorhaben zur TNF-a-Inhibition prämiert}

Anlässlich der Forschungsförderung Inflammation vergab Pfizer im November 2014

120.000 Euro an zwei Projekte aus dem Bereich Dermatologie.

Preisträger der Förderpreise, die während des DGRh-Kongresses in Düsseldorf bekannt gegeben wurden, sind Dr. Detlef Becker-Capeller, Stade, und PD Dr. Thomas Herzinger, München.

Ausgewählt wurden die Projekte in anonymisierter Form von den international renommierten und unabhängigen Experten Prof. Dr. James Krueger, New York, und Prof. Dr. Jonathan Barker, London.

Dr. Detlef Becker-Capeller, Stade, erhielt die Ehrung für das Projekt:, Standardisierte Untersuchung ausgewählter Enthesen, Sehnen und Kapillaren zur Frühdiagnostik der PsA bei Patienten mit neu entdeckter Psoriasis vulgaris", PD Dr. Thomas Herzinger, Klinik und Poliklinik für Dermatologie und Allergologie, München, wurde für das Projekt: „Secondary prevention of sunburn with TNF alpha antagonists" ausgezeichnet.

www.pfizer.de 